\title{
Response bias in very first judgments of the reflectance of grays: Numerical versus linear estimates
}

\author{
E, C. POULTON AND D. C. V. SIMMONDS, APPLIED PSYCHOLOGY RESEARCH UNIT, CAMBRIDGE, ENGLAND
}

R. M. WARREN, UNIVERSITY OF WISCONSIN

Three hundred unsophisticated Os judged the lightness of a gray, reflectance $44 \%$, against a white, reflectance $83 \%$. Half gave a numerical estimate calling the white 100. The remainder marked a $100 \mathrm{~mm}$ line labeled 100 at one end and zero at the other. Both distributions of estimates were skewed with a mode around 60 . The linear estimates tended to avoid the middle of the line $(p<.01)$. The numerical estimates tended to end in zero or five $(p<.001)$. The two distributions were not quite reliably different, but the medians of subgroups of 10 Os drawn from the two distributions were reliably different $(p<.05)$.

It has recently become clear (Poulton, 1967a) that estimations of sensory magnitude, like other kinds of decisions (Broadbent \& Gregory, 1963), can be influenced by response bias. Independent va riables on the input side which interact with response bias are the range of stimulus magnitudes presented (Poulton, 1967b), the position of the standard within the range, and the distance of the first variable from the standard (Poulton \& Simmonds, 1963). When the responses are numerical estimates, response bias also interacts with the size of the modulus, or number given to the standard (Poulton \& Simmonds, 1963), and with whether the set of numbers is finite or infinite (Poulton, Simmonds, Warren \& Webster, 1965). A third source of interaction on the response side, investigated here, is whether magnitude estimates are given in digital or analog form.

The pioneer in the field of magnitude estimation, (Richardson, 1929), used both numerical and linear estimates in different experiments. But he did not compare the two methods; he took both sets of data at their face value. The first experimenters to compare the two methods appear to have been Warren and Poulton (1960, Table 1). They used separate groups of 50 unsophisticated observers (Os). The display consisted of white and gray rectangular strips, each $11-1 / 4 \mathrm{x}$ 8-7/8 in., with one of the longer edges of each strip contiguous with the other. The white paper had a reflectance of $85 \%$, and the gray $8 \% 19.43 \%$ relative to the white). For the numerical estimates the white was called "100", and $O$ was asked for the smaller number describing the lightness of the gray. For the linear estimates a $100 \mathrm{~mm}$ line was labeled "white" at one end and "black" at the other; $O$ had to mark the position on the line corresponding to the reflectance of the gray patch. The numerical estimates were found to have a median of 30 and a mean of 29 . The median of the marks was $34 \mathrm{~mm}$ from the end of the line labeled "black," the mean $38 \mathrm{~mm}$. A reanalysis of the data indicates that the difference between the two distributions is reliable at the .01 level on a Mann-Whitney $U$ test (Siegel, 1956, p. 116). Examination of the two distributions showed that both had about the same number of estimates $>40$ (15 and 16 estimates respectively). But there were only two linear estimates within the

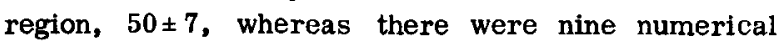
estimates, six of them at 45. This left eight linear estimates greater than 60 , compared with only one numerical estimate.

The present experiment was undertaken to investigate this phenomenon, making the two tasks as comparable as possible. Instead of labeling the ends of the line "white" and "black," they were labeled "100" and "zero." A variable was chosen which had a reflectance well away from the dark end of the range of grays, since the judgments of dark variables are more influenced by adaptation-level effects than are the judgments of variables with intermediate reflectances (Poulton, 1967a). What $O$ decides to take as black in estimating the relative reflectance of grays plays a role somewhat comparable to the level of dark adaptation in making brightness judgments (S.S. Stevens, 1958).

\section{Methods}

The 300 Os were mainly male undergraduates of Cambridge University, although a few graduates and women students were included. Those who reported that they had attended lectures in psychology, or had served in a similar experiment in the past, were excluded.

The display consisted in a piece of white paper $17.5 \mathrm{x}$ $11.3 \mathrm{in}$. with a reflectance of $83 \%$. It lay on a table directly in front of $O$, arranged symmetrically with a long side closest to him. For the Os making linear estimates, it was labeled 100 in pencil on the side nearest to the end of the line which was labeled 100. In the middle of the white paper was placed a gray stimulus patch $2.0 \times 1.5 \mathrm{in}$. with its long side parallel to the long side of the white paper. It had a reflectance of $44 \%$. The white paper and patch were coated with nonglossy paint. As in the previous experiments (Warren \& Poulton, 1960; Poulton \& Simmonds, 1963; Poulton et al, 1965) the lighting on the table was at the ambient level to which $O$ had become adapted. 


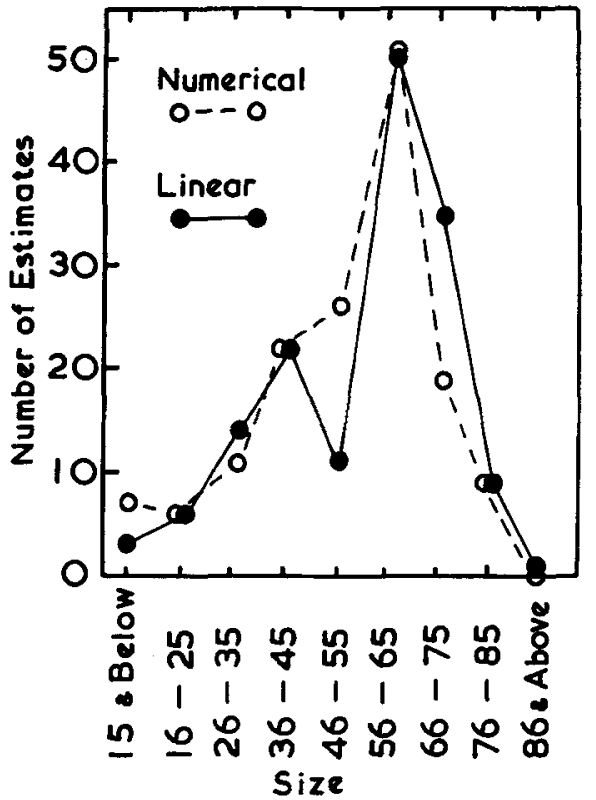

Fig. 1. Distributions of first numerical and linear estimates grouped by 10s. Each function is for a separate group of $150 \mathrm{Os}$.

The procedure followed that of previous experiments carried out in Cambridge colleges. For the numerical estimates $O$ had a white answer slip measuring $4 \times 3$ in. A white card measuring $8 \times 5$ in. had the following instructions typed on it "You will see a gray patch of paper lying on a white blotter. If the lightness of the blotter is called 100, what (smaller) number describes the lightness of the gray patch? Please write down your answer on the slip of paper provided." The instructions were explained if necessary by paraphrasing them. After $O$ had written down his numerical estimate, the gray patch was removed and replaced by a patch with a different reflectance. The $O$ was required to judge this also, in order to check that he was treating the white background as 100 and not as zero. The results of Os who apparently inverted the number scale were discarded. Data from 150 Os were used.

For the linear estimates $O$ had an answer slip measuring $8 \times 5 \mathrm{in}$. which carried a horizontal line $100 \mathrm{~mm}$ long labeled 100 at one end and zero at the other. For half the Os the 100 was at the left end; for the other half it was at the right end. The instructions ran as follows "You will see a gray patch of paper lying on a white blotter. A line will be given you marked "100" at one end and "zero" at the other. Consider this to represent linearly the numbers lying between 100 and zero. If the 100 represents the lightness of the white blotter, please place a mark on the line to indicate the (smaller) number representing the lightness of the gray patch." After O had placed his mark on the line, the answer slip was turned over to reveal another identical line $100 \mathrm{~mm}$ long, and the gray patch was changed. The second judgment was employed as a check that $O$ was treating the white background as 100 and not as zero. As before data from 150 Os were used. The position of each mark was measured to the nearest $1 \mathrm{~mm}$ from the end of the line labeled zero, and this measure was taken as the linear estimate.

\section{Resulis}

Figures 1 and 2 illustrate the differences between the distributions of the first responses made under the two conditions. Figure 1 shows that the linear estimates tended to avoid the middle of the line. When the estimates were dichotomized as between 46 and 55 or outside these limits, chi squared showed that the difference between the two distributions was reliable at better than the .01 level of confidence. Comparing the two distributions, the linear estimates which would have fallen in the region of 50 appear to have been shifted upwards to the region of 70 .

Figure 2 shows that the first numerical estimates tended to end in zero, and to a lesser extent in five. When the second digit of each estimate was placed in one of four categories: zero, between one and four, five, or between six and nine, chi squared showed that the difference between the two distributions was reliable at well beyond the .001 level. The numerical estimates can be said to have a coarser grain than the linear estimates.

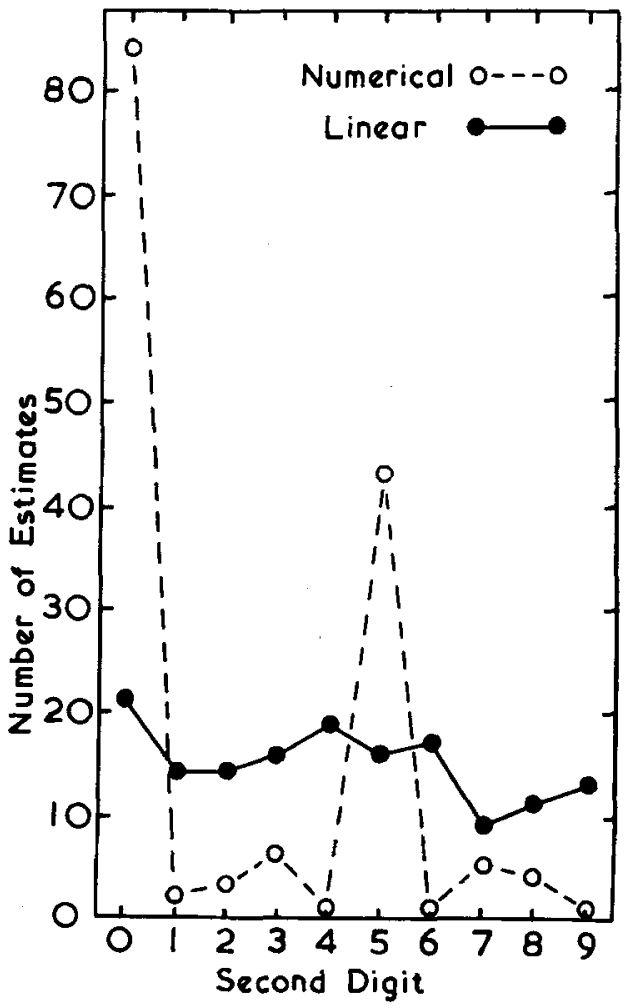

Fig. 2. Distributions of first numerical and linear estimates grouped according to the value of the second digit. Each function is for a separate group of $150 \mathrm{Os}$ 
Table 1. Differences between numerical and linear estimates

\begin{tabular}{lccr} 
Groups of 150 Os & Numerical estimates & Linearestimates & $P$ \\
\hline $\begin{array}{l}\text { Range } \\
\text { Medion }\end{array}$ & $4-85$ & $14-86$ & .06 \\
(Mean & 60 & 61 & .10 \\
(SE & 53.0 & 55.1 & \\
(Geometric Mean & 1.41 & 1.35 & .08 \\
(SE & 48.7 & 52.0 & \\
(SE & 1.03 & 1.03 & \\
Medians of 15 groups, each of 10 & 05 & & \\
\hline Range & $38.3-68.3$ & $49.5-64.0$ & .04 \\
Medion & 56.4 & 61.1 & .04 \\
(Mean & 56.6 & 60.5 & \\
( & 1.87 & 1.14 & \\
(SE & 56.2 & 60.3 & .03 \\
(Geometric Mean & 1.04 & 1.02 & \\
( & &
\end{tabular}

The top half of Table 1 shows how the differences between the two distributions affected the medians, the arithmetic and geometric means, and their standard errors. None of the differences between the two groups of 150 Os was quite reliable statistically. The median numerical estimate only just reached 60 ; small changes in the estimates made by six Os would have reduced it to 55, owing to the tendency of the numerical estimates to end in zero or five (Figure 2). On a one-tailed MannWhitney $U$ test (Siegel, 1956) the two distributions were nearly reliably different at the .05 level of confidence.

The bottom half of the table compares the effects which the two distributions had upon the medians of randomly-selected subgroups of $10 \mathrm{Os}$, which is about the usual size for experiments on magnitude estimation. The 15 medians of the subgroups making linear estimates tended to lie above the 15 medians of the subgroups making numerical estimates. Whereas 11 out of the 15 linear medians lay above 60 , only three of the 15 numerical medians exceeded 60. The two sets of medians were reliably different on a one-tailed Mann-Whitney $U$ test. The means and geometric means of the two sets of medians were reliably different on one-tailed $t$ tests.

\section{Discussion}

The results illustrate the effects of yet another constraint which the experimenter has to impose upon $O$ in order to obtain magnitude estimates: the way in which the responses are made. When asked for a fractional estimate using the numbers from 100 to zero, O's first estimate is likely to end in zero or five. This probably reflects his initial uncertainty as to the exact numerical value to choose. It increases the coarseness of the grain of the data. Presumably a finer grain distribution of estimates could be obtained by instructing $O$ s to estimate to the nearest 10 or five, and then to select a last digit if necessary at random. This is analogous to the forced-choice technique threshold measurements, as contrasted with allowing $O$ a third "uncertain" category. To the authors' knowledge it has not yet been used in magnitude estimation.

If instead of being asked for a number between 100 and zero, $O$ is asked to mark a line, his first judgment tends to avoid the middle of the line. This tendency also could presumably be prevented by special instructions. But here it would be extremely difficult to ensure that the instructions did not contain their own bias. For example, the warning: "Don't avoid the middle of the line if this is where you think the mark should go." might encourage $O$ to believe that the middle was the correct position.

It could be objected that these effects are likely to be small and transient, and that they could be excluded from the data by giving $O$ a few practice judgments before starting the experiment. However, there is now considerable evidence to suggest that $O$ 's very first judgment may be his most valid judgment (Poulton, 1967a), since it is unbiased by transfer effects (Poulton \& Freeman, 1966). The distortions introduced into first judgments by asking for a numerical or a linear estimate are small compared with the distortions which can be introduced into subsequent judgments by transfer effects.

\section{References}

Broadbent, D. E., \& Gregory, M. Vigilance considered as a statistical decision. Brit. J. Psychol., 1963, 54, 309-323.

Poulton, E. C. The new psychophysics: six models for magnitude estimation. Psychol. Bull., 1967a, in press.

Poulton, E. C. Population norms of top sensory magnitudes and S. S. Stevens' exponents. Percept. \& Psychophys., 1967b, 2 , 312-316.

Poulton, E. C., \& Freeman, P. R. Unwanted asymmetrical transfer effects with balanced experimental designs. Psychol. Bull., 1966, 66, 1-8.

Poulton, E. C., \& Simmonds, D. C. V. Value of standard and very first variable in judgments of reflectance of grays with various ranges of available numbers, J. exp. Psychol., 1963, 65, 297304.

Poulton, E. C., Simmonds, D. C. V., Warren, R. M., \& Webster, I. C. Prior context and fractional versus multiple estimates of the reflectance of grays against a fixed standard. J. exp. Psychol., $1965,69,496-502$.

Richardson, L. F. Quantitative mental estimates of light and colour. Brit. J. Psychol., 1929, 20, 27-37.

Siegel, S. Nonparametric statistics for the behavioral sciences. New York: McGraw-Hill, 1956.

Stevens, S. S. Some similarities between hearing and seeing. The Laryngoscope, 1958, 68, 508-527.

Watren, R. M., \& Poulton, E. C. Basis for lightness-judgments of grays. Amer. J. Psychol., 1960, 73, 380-387.

\section{Note}

1. P. M. E. Altham kindly advised on the statistics, and C. H. Brock helped to calculate the geometric means. The work was supported in part by the British Medical Research Council, and in part by the Air Force Office of Scientific Research of the Air Research and Development Command, U.S.A.F., through its European Office.

(Accepted for publication October 15, 1967.) 